\title{
Parallel and Distributed Computing in Multiple Images- A Combined Restoration
}

\author{
Sudha Mishra ${ }^{1}$, Sandeep Sahu ${ }^{2}$, Naazish Rahim ${ }^{3}$ \\ ${ }^{1}$ Software System In Department of Computer Science \\ SRIT, Jabalpur, M.P. \\ ${ }^{2}$ Software System In Department of Computer Science \\ SRIT, Jabalpur, M.P. \\ ${ }^{3}$ In Department of Computer Science \& Engineering \\ CEC, Bilaspur, C.G.
}

\begin{abstract}
Image restoration is the process of recovering the original image from the degraded or noisy image. We are using multiple images for processing, the identifier select the blur image for further process and restore after applying the algorithms. Our object is identifying the blur image and restores them by parallel distributed computing. We are using blind deconvolution algorithm for restoration process. In which PSF value is unknown that is the intensity value by which image is blurred. We are applying 3 approaches first one is blind deconvolution algorithm. Second is parallel computing and third one is distributed computing. In parallel computing approaches one local scheduler is working for distributing the job and restore result at once. In distributed computing each worker has their same program after running program send the result to the job manager. This overall architecture is depended upon the parallel distributed computing. Job manager handled the overall process done by the worker.
\end{abstract}

Keywords: Blind Deconvolution Algorithm,Degradation Model, PSF, job scheduler.

\section{Introduction}

Blind Deconvolution Algorithm is the process of denoising the image that has been blurred by any reason. It can be used effectively when no information of noise/distortion is known and restores image and PSF value simultaneously. Blind deconvolution is the recovery of a blurred image when the blur kernel is unknown. The Blind Deconvolution Algorithm can be used effectively when no information about the distortion or blurring and noise is known. The algorithm restores the image and the point-spread function (PSF) simultaneously. The blurring, or degradation, of an image can be caused by many factors such as movement during the image capture process, by the camera or, taking more times by capturing process, by the Out-of-focus optics, use of a wide angle lens, scattered light, and distortion in confocal microscopy. Image deblurring is an inverse problem of linear degradation. The distributed system consists of multiple computers that can communicate through a local computer network the overall function is an autonomous. The computers interact with each other in a network for achieving a common goal by the local scheduler. A computer program that runs in a distributed system or processor is called a distributed program, and distributed programming. Distributed computing also refers to the use of distributed systems to solve computational problems. In distributed computing, a problem is divided into many tasks, each of which is solved by one or more computers. A distributed computing or system may have a common goal, such as solving a large computational problem such problems that may be work on a same time. In this method each computer may have its own user with their sub function. The purpose of the distributed system is distributing the sub function into their worker. Job scheduler provides communication services to their worker.

The worker can use shared resources in their local communication area. In parallel computing, all workers have a shared memory. Shared memory can be used to exchange information between processors. In distributed computing, each processor has its own private memory (distributed memory) that is shared. Information is exchanged by passing different types of messages between the processors.

\section{Related Work}

There is no of proposed method in Blind deconvolution algorithms in signal and image processing in literature surey, a few considers [1], [9], [10], and [11]. In [2], Ramya et al concede the problem with inference of the known parameters PSF, Blur length, Blur angle and type of noise, needed for blurring an image. First they apply their degradation model on the input image and use canny filter to detect the edges from blurred image, but in a single image. The iterative Blind deconvolution algorithm was first proposed by Ayers and Dainty in 1988 [8]. In [7], Li et al, proposed statistical method is proposed for deblurring two-tone images, i.e., images with two unknown grey levels that are blurred by an unknown linear filter. The key idea of the proposed method is to adjust a deblurring filter until its output becomes two tones. Two optimization criteria are proposed for the adjustment of the deblurring filter. The various approaches that have appeared in the literature depend upon the particular degradation and image models [3], [4]. In [5] R. Fergus et al, describes the different features of removing camera shake from a single photograph. The heterogeneous process is done in [6]. It describes the distributed programming model. There is wide variety of proposed work but we are using blur identifier for restoration process. In a digital camera possibility is some picture may be blur. Our object is that choosing those images that have been blurred and send to the local scheduler for processing into their worker.

\section{Proposed Method}

In distributed computing each worker has individual memory and parallel computing memory is shared. Our method is performing the same operations on a set of data. The operation is sequence of operation for deblurring the process. 
Each worker working on a separate piece of the set. Distributed computing is the method of communicating and maintaining the work among multiple workers.

We are simulating this one on data parallel programming model. By this we can perform the same task in in 4 local machine. We will able to show that the algorithm design and analysis techniques developed for the task/channel model apply directly to data-parallel programs; in particular, they provide the concepts required to understand the locality and scalability of data-parallel programs.

\subsection{Characteristics}

(a) Saves time is wall clock time is fixed for continuation of process,

(b) Cost savings,

(c) It can improve the computing communication is data exchange between parallel tasks,

(d) Synchronization is the coordination of parallel tasks in real time,

(e) CPU performance,

(f) Memory that may be shared,

(g) Latency for equalization,

(h) Bandwidth and

(i) Homogeneous task

\subsection{Data Parallel Programming model}

In the data parallel programming model, a computation is defined in terms of a sequence of instructions executed on multiple elements of a memory object using distributed computing toolbox. Data Parallelism Divide the dataset into grids or sectors and solve each sector on a separate execution unit. We are using divide and conquer method using SIMD architecture.

\subsection{Task Parallel Programming model}

Task-Parallel Programming Model is used for large data set it can divide the problem into different tasks and execute the tasks on different units. In this model, a kernel instance is executed independent of any index space. This is equivalent to executing a kernel on a computer device with a work-group and NDRange containing a single work-item. Parallelism is expressed using vector data types implemented by the device, enqueuing multiple tasks, and/or enqueuing native kernels developed using a programming model orthogonal to OpenCL. Task parallelism is also known as function parallelism and control parallelism that is a form of parallelization of computer code across multiple processors in parallel computing environments. Task parallelism focuses on distributing execution processes (threads) across different parallel computing nodes.

\subsection{Synchronization}

Synchronization is must in homogeneous task. For performing the same task in parallel distributed computing at a same time synchronization of data is must.

Capacity vs. Capability:

Creating large supercomputers to facilitate large throughput of small parallel jobs, cheaper, slower interconnects Clusters running Linux, OS X, or Windows, Easy to build. In Capability computing creating large supercomputers to enable computation on large scale, running the entire machine to perform one task, good fast interconnect and balanced performance important, usually specialized hardware and operating systems.

\subsection{Network}

The performance of a distributed memory architecture is highly dependent on the speed and quality of the interconnect.

\section{Latency}

The time to send a 0 byte packet of data on the network. Time taken by sender sending actual information to the local scedular.

\section{Bandwidth}

The rate at which a very large packet of information can be sent. In parallel distributed computing we are using multiple images.

\section{Topology}

The configuration of the network that determines how many processing units are directly connected.

\section{Parallelism}

Parallelism performing multiple tasks at once and can distribute the work into multiple execution units.

We are using simple method available in [11]. Data parallel model for the process the process is same as deblurring but work is distributed by the data manager.

\section{Overall Process}

The process of running your program in parallel now requires some steps:

Step I: Call Multiple Images.

Step II: Call Identifier for selecting the blur images in multiple images for further processing.

Step III: Call Local Scheduler for distributing the job.

Step IV: By Local Scheduler request a number of workers.

Step V: Filter the image.

Step VI: Apply Blind Deconvolution Algorithm.

Step VI: Issue the normal command to run the program. The client program will call on the workers as needed.

Step VI: Released the worker.

Step VII: Store images after processing deblurred image.

Step VII Final result.

Step VII Display Image.

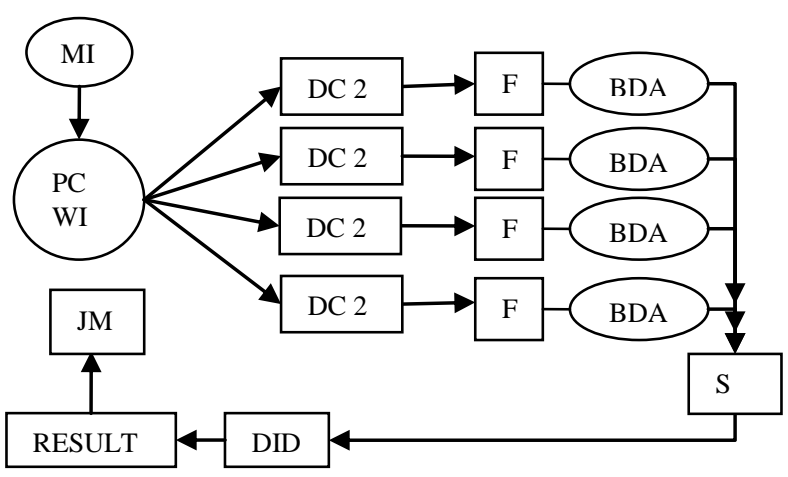

Figure 1. Overall Process 
In above diagram it represents the parallel distributed computing identifying the blur image between multiple images.

MI- multiple images, BDA- Blind Deconvolution

Algorithm, PCWI-Parallel computing with identifier,

DC- Distributed computing with 4 workers, F-Filter, S-Shared Memory, DID-Display image with database, RESULT-Result is final computed result, JM-Job Manager display the result.

Table 1.Result Table

\begin{tabular}{|l|l|l|l|l|}
\hline Work & Latency & $\begin{array}{l}\text { Networ } \\
\mathrm{k}\end{array}$ & $\begin{array}{l}\text { Topolog } \\
\mathrm{y}\end{array}$ & $\begin{array}{l}\text { Synchronou } \\
\mathrm{s}\end{array}$ \\
\hline Serial & $4.34 \mathrm{~s}$ & Large & 12 & No \\
\hline Parallel & $2.2 \mathrm{~s}$ & Small & 8 & Less \\
\hline $\begin{array}{l}\text { Distribute } \\
\mathrm{d}\end{array}$ & $1.5 \mathrm{~s}$ & Small & 6 & More \\
\hline Group & $1.2 \mathrm{~s}$ & Small & 4 & More \\
\hline
\end{tabular}

\section{Conclusions}

This paper is mainly focused on identifying those images that have been blurred between multiple images. The goal of this paper is to analyze the problem blurring process and improving the algorithms. In which multiple works done in one time in parallel distributed computing same task is divided into their worker for parallel computing. Job scheduler maintains the synchronization of work. Parallel computing is fast. Each processor has its own memory. We want to perform homogeneous task for the same. Multiple jobs can run their sub applications on the server simultaneously.

\section{References}

[1] G. R. Ayers and J. C. Dainty, "Interative Blind Deconvolution Method and Its Applications, "Optics Letters, vol. 13, pp. 547-549, 1988.
[2] Ramya, S.; Mercy Christial, T, "Restoration of Blurred Images using Blind Deconvolution Algorithm," IEEE, on Emerging Trends in Electrical and Computer Technology (ICETECT), pp.496 -499,2011

[3] A. K. Katsaggelos, ed., Digital Image Restoration. New York Springer-Verlag, 1991.

[4] H. C. Andrews and B. R. Hunt, Digital Image Restoration. New Jersey: Prentice-Hall, Inc., 1977.

[5] R. Fergus, B. Singh, A. Hertzmann, S.T. Roweis, and W.T. Freeman, "Removing Camera Shake from a Single Photograph,"Proc. ACM SIGGRAPH, 2006.

[6] Yuan Shi "A Distributed Programming and It's Application to Computation Intensive Problems for Heterogeneous Environmets" AIP Conference Proceedings 283, Earth and Space Science Information Systems, Pasadena, CA 1992, Editor: Arthur Zygielbaum, pp. 827848 .

[7] Ta-Hsin Li, Member, IEEE, and Keh-Shin Lii, Member, IEEE, IEEE TRANSACTIONS ON IMAGE PROCESSING, VOL. 11, NO. 8, AUGUST 2002.2367.

[8] A.K. Katsaggelos and K.T. Lay, Likelihood Blur Identification Restoration Using the EM Algorithm,'IEEE Trans. Signal Processing, vol. 39, no. 3, pp. 729- 733, Mar. 1991.

[9] A.C. Likas and N.P. Galatsanos, "A Variational Approach for Bayesian Blind Image Deconvolution," IEEE Trans. Signal Processing, vol. 52, no. 8, pp. 2222-2233, Aug. 2004.

[10 R. Molina, A.K. Katsaggelos, J. Abad, and J. Mateos, "A Bayesian Approach to Blind Deconvolution Based on Dirichlet Distributions," P Int'l Conf. Acoustics, Speech, and Signal Processing.1997.

[11 D. Kundur and D. Hatzinakos, "Blind Image Deconvolution,"IEEE Signal Processing Magazine, vol. 13, no. 3, pp. 43-64, May 1996. 